\title{
Effective interactions, structure, and isothermal compressibility of colloidal suspensions
}

\author{
Marjolein Dijkstra ${ }^{\text {a) }}$ \\ Debye Institute, Condensed Matter Physics, Utrecht University, Princetonpln 5, 3584 CC Utrecht, \\ The Netherlands \\ René van Roij \\ Institute for Theoretical Physics, Utrecht University, Princetonpln 5, 3584 CC Utrecht, The Netherlands \\ Robert Evans \\ H. H. Wills Physics Laboratory, University of Bristol, Tyndall Avenue, Bristol BS8 1TL, United Kingdom
}

(Received 25 February 2000; accepted 21 June 2000)

\begin{abstract}
We study the effective interactions, structure, and the isothermal compressibility of a binary mixture interacting with pairwise additive pair potentials. By integrating out the degrees of freedom of species 2 in the partition sum we first show that a binary mixture can be mapped formally onto an effective one-component system with an effective Hamiltonian consisting of a structure-independent term, which contributes to the total pressure and chemical potential of the system, but does not affect the phase behavior, and a structure-dependent potential of mean force, which contains pair-, triplet-, and higher-body interactions. We then show that the 1-1 structure factor and pair correlation function, and the total isothermal compressibility of the mixture are equal to those of the effective one-component system, provided the mapping is exact. We illustrate and confirm these results by calculating the structure factors and pair correlation functions of the binary AsakuraOosawa model, which is a simple model for colloid-polymer mixtures, and those of the corresponding one-component system for a size ratio such that the mapping onto an effective one-component Hamiltonian with a strictly pairwise potential of mean force is exact. The distinction between the osmotic and total compressibility of the mixture is emphasized. (C) 2000 American Institute of Physics. [S0021-9606(00)51335-X]
\end{abstract}

\section{INTRODUCTION}

Colloidal suspensions are complex fluids that consist of mesoscopic particles suspended in a solvent (e.g., water). Often other components, such as salt ions or polymers, are present as well. Such multicomponent fluids are difficult to describe as very different length and time scales are involved for the different constituents. This problem is often circumvented by coarse graining, i.e., by eliminating the degrees of freedom of the smaller particles and the solvent, which yields a one-component system that is described by effective interactions. The ideal-gas version of this concept dates back to Van't Hoff, who showed that the osmotic pressure of a suspension is proportional to the solute concentration. The formalism for describing the more complicated and interesting case of interacting, nonideal particles was introduced by McMillan and Mayer in 1945. An important ingredient of the McMillan-Mayer solution theory is the so-called "potential of mean force,' which plays the role of the interaction Hamiltonian in the effective one-component system. If the potential of mean force is assumed to be pairwise additive, with some specific effective pair-potential (e.g., the DLVO potential in the case of charge-stabilized colloidal suspensions, ${ }^{2}$ or the depletion potential in the case of colloid-polymer, colloid-colloid mixtures, ${ }^{3,4}$ and mixtures

${ }^{a)}$ Electronic mail: m.dijkstra@phys.uu.nl of micelles and emulsion droplets ${ }^{5}$ ) one can determine the phase behavior of the colloidal system via simulation or theory using the same techniques as for one-component systems. It is known that predictions for the phase behavior of charge-stabilized suspensions which follow from the effective DLVO theory are in agreement with experiments, at least at sufficiently high salt concentrations. ${ }^{2,6}$ The status of the DLVO theory at very low salt concentrations is more controversial, but recent theoretical studies of effective interactions can explain some of the features of the experimentally observed phase behavior. ${ }^{7}$ In the case of very asymmetric binary hard-sphere mixtures, we compared the phase behavior determined from simulations of the effective onecomponent system with that from simulations of the true binary mixture. ${ }^{8}$ The agreement we found was surprisingly good, and provides important justification for the use of the pairwise depletion potential in the effective one-component system. ${ }^{8}$ Thus, it is fair to argue that the concept of integrating out or coarse graining has been successful in describing phase diagrams of colloidal systems.

An important subsequent question is whether the equilibrium structure and the various thermodynamic properties of the mixture are also well-described by the potential of mean force or the effective Hamiltonian. Some recent examples in the literature suggest that this is not the case. Recent experiments on charge-stabilized colloidal crystals, for instance, show that the bulk modulus-the inverse of the 
compressibility - as determined from the colloid structure factor at long wavelengths is three times smaller than that calculated from the DLVO theory. ${ }^{10}$ The authors then conclude that the conventional DLVO theory for the effective colloidal interactions fails to account for the elastic properties of colloidal crystals. ${ }^{10}$ Another example is given by Louis et al., who calculate the structure factors of the Asakura-Oosawa model for colloid-polymer mixtures in the Percus-Yevick (PY) approximation. ${ }^{11}$ They find that the isothermal compressibility $\chi_{T}$ obtained from the $k \rightarrow 0$ limit of the partial structure factors [see Eq. (9)] in the twocomponent Asakura-Oosawa model is smaller by an order of magnitude than $\chi_{T \text {,eff }}$, the osmotic compressibility, obtained from the $k \rightarrow 0$ limit of the colloidal structure factor of the corresponding effective one-component system [see Eq. (13) $]^{11}$

In this article we address the issue of how to compare the thermodynamic properties of the mixture with those of the effective one-component system. Making such comparisons is not trivial. We first show that when the mapping of the homogeneous mixture onto the corresponding effective one-component system is exact, the colloid-colloid structure in the mixture should be equal to the colloidal structure in the effective one-component system. In particular this implies that the pair correlation functions and structure factors of the colloids obtained from the effective one-component Hamiltonian description are exactly equivalent to the colloid-colloid pair correlation functions and structure factors in the colloidal suspension. The same equivalence holds for higher-order correlations. We then demonstrate that thermodynamic properties, focusing on the isothermal compressibility, are also equal in the mixture and in the corresponding effective one-component system-provided once again the mapping is exact and the comparison is made at precisely the same state point. As an example we calculate the structure factors and pair correlation functions of the two-component Asakura-Oosawa model and those of the effective onecomponent system for a polymer/colloid size ratio such that the mapping onto an effective one-component Hamiltonian, whose potential of mean force has strictly pair interactions, is known to be exact. ${ }^{9}$ We show that the structure factors and the pair correlation functions are equal in the two corresponding systems. $\chi_{T}$ as calculated from the $k \rightarrow 0$ limit of the partial structure factors of the mixture does agree with the full result for the effective one-component system. The main contribution to the latter is an ideal polymer term not contained in $\chi_{T \text {,eff }}$ and not considered in Ref. 11.

\section{MAPPING A MIXTURE ONTO AN EFFECTIVE ONE- COMPONENT SYSTEM}

We showed in Ref. 8 that a homogeneous binary mixture can be formally mapped onto an effective one-component system with effective interaction Hamiltonian $H^{\text {eff }}$ by integrating out the degrees of freedom of one of the two species in the partition sum. For completeness we briefly recapitulate. We consider a classical fluid of two species, label 1 and 2 , in a macroscopic volume $V$. For particle numbers $N_{1}$ $=V \rho_{1}$ and $N_{2}=V \rho_{2}$, the total Hamiltonian consists of (trivial) kinetic energy contributions and the interaction
Hamiltonian $H=H_{11}+H_{12}+H_{22}$. We assume pairwise additive pair potentials $\phi_{11}, \phi_{12}$, and $\phi_{22}$ in the mixture (but not in the effective one-component system). Thus, $H_{11}$ $=\Sigma_{i<j}^{N_{1}} \phi_{11}\left(\mathbf{R}_{i j}\right), \quad H_{12}=\sum_{i=1}^{N_{1}} \Sigma_{j=1}^{N_{2}} \phi_{12}\left(\mathbf{R}_{i}-\mathbf{r}_{j}\right), \quad$ and $H_{22}$ $=\Sigma_{i<j}^{N_{2}} \phi_{22}\left(\mathbf{r}_{i j}\right)$, where $\mathbf{R}_{i}$ and $\mathbf{r}_{j}$ denote the coordinates of the particles of species 1 and 2, respectively, $\mathbf{R}_{i j}=\mathbf{R}_{i}-\mathbf{R}_{j}$ and $\mathbf{r}_{i j}=\mathbf{r}_{i}-\mathbf{r}_{j}$. It is convenient to consider the system in the $\left(N_{1}, z_{2}, V\right)$ ensemble, in which the fugacity $z_{2}$ of species 2 is fixed. Omitting the explicit $T$ dependence, we can write the thermodynamic potential $F\left(N_{1}, z_{2}, V\right)$ of this system as $\exp [-\beta F]=\operatorname{Tr}_{1} \exp \left[-\beta H^{\mathrm{eff}}\right]$, where $H^{\text {eff }}=H_{11}+\Omega$ is the effective Hamiltonian of species 1 and $\beta=1 / k_{B} T$. Here, $\Omega$ $=\Omega\left(N_{1}, z_{2}, V ;\{\mathbf{R}\}\right)$ is the grand potential of the fluid of species 2 in the external field of a fixed configuration of $N_{1}$ particles of species 1 with coordinates $\left\{\mathbf{R}_{i}\right\} ; i=1,2, \ldots N_{1}$, and is given by $\exp [-\beta \Omega]=\sum_{N_{2}=0}^{\infty} z_{2}^{N_{2}} \operatorname{Tr}_{2} \exp \left[-\beta\left(H_{12}\right.\right.$ $\left.\left.+H_{22}\right)\right]$. The trace $\operatorname{Tr}_{\nu}$ is short for $1 / N_{\nu} ! \Lambda_{\nu}^{3 N_{v}}$ times the volume integral over the coordinates of all the particles of species $\nu . \Lambda_{\nu}$ is the thermal wavelength.

By performing a Mayer cluster expansion, we then showed that $\Omega$ can be written as $\sum_{n=0}^{N_{1}} \Omega_{n}$, where $n$ labels the number of particles of species 1 that interact simultaneously with the "sea" of species 2 . The zero-body term $\Omega_{0}$ is the grand potential of a pure system of species 2 at fugacity $z_{2}$ in a volume $V$, i.e., $\Omega_{0}\left(z_{2}, V\right)=-p_{2}\left(z_{2}\right) V$, with $p_{2}\left(z_{2}\right)$ the pressure of the reservoir of species 2 . The one-body term is of the form $\Omega_{1}\left(N_{1}, z_{2}\right)=N_{1} \omega_{1}\left(z_{2}\right)$, with $\omega_{1}\left(z_{2}\right)$ the grandpotential difference between a "sea" of species 2 at fugacity $z_{2}$ with and without a particle of species 1 at the origin. Within the same formalism we obtain $\Omega_{2}\left(N_{1}, z_{2} ;\{\mathbf{R}\}\right)$ $=\Sigma_{i<j}^{N_{1}} \omega_{2}\left(z_{2} ; R_{i j}\right)$, where $\omega_{2}$ is the grand potential difference between a "sea" of species 2 at fugacity $z_{2}$ containing two particles of species 1 separated by a finite distance $R_{i j}$ $=\left|\mathbf{R}_{i}-\mathbf{R}_{j}\right|$ and by infinite distance. Similar expressions can be given for the three- and higher-body terms, e.g., $\Omega_{3}$ $=\Sigma_{i<j<k}^{N_{1}} \omega_{3}\left(z_{2} ; R_{i j k}\right)$. Thus, we arrive at the effective onecomponent Hamiltonian

$$
\begin{aligned}
H^{\mathrm{eff}}\left(N_{1}, z_{2}, V ;\{\mathbf{R}\}\right)= & -p_{2}\left(z_{2}\right) V+N_{1} \omega_{1}\left(z_{2}\right) \\
& +W\left(N_{1}, z_{2} ;\{\mathbf{R}\}\right),
\end{aligned}
$$

where the dependence on the coordinates $\{\mathbf{R}\}$ resides only in the potential of mean force

$$
\begin{aligned}
W\left(N_{1}, z_{2} ;\{\mathbf{R}\}\right)= & \sum_{i<j}^{N_{1}}\left[\phi_{11}\left(R_{i j}\right)+\omega_{2}\left(z_{2} ; R_{i j}\right)\right] \\
& +\sum_{i<j<k}^{N_{1}} \omega_{3}\left(z_{2} ; R_{i j k}\right)+\ldots .
\end{aligned}
$$

Consequently, the thermodynamic potential of interest $F$ can be written as

$$
F\left(N_{1}, z_{2}, V\right)=-p_{2}\left(z_{2}\right) V+N_{1} \omega_{1}\left(z_{2}\right)+A\left(N_{1}, z_{2}, V\right),
$$

with $A$ defined by $\exp [-\beta A]=\operatorname{Tr}_{1} \exp [-\beta W]$, i.e., $A$ is the Helmholtz free energy of a one-component system with an interaction Hamiltonian that equals the potential of mean force. 
A few remarks are in order. (i) Although the problem of the binary mixture has been reduced formally to that of an effective one-component fluid, one must determine the effective potentials $\omega_{n}$ for $n=1,2 \ldots$ before explicit calculations of $F$ and $A$ can be made. (ii) Since the terms $\Omega_{0}$ $=-p_{2}\left(z_{2}\right) \mathrm{V}$ and $\Omega_{1}=N_{1} \omega_{1}\left(z_{2}\right)$ in the effective Hamiltonian in Eq. (1) do not depend on the instantaneous coordinates $\{\mathbf{R}\}$ of the particles of species 1 , they do not affect the structure of the effective one-component system, as we will see in more detail below. Moreover, these two terms do not affect the phase behavior of the two-component system because of their trivial $V$ and $N_{1}$ dependence, which gives rise to innocuous shifts in the pressure $p=-(\partial F / \partial V)_{N_{1}, z_{2}}$ and chemical potential $\mu_{1}=\left(\partial F / \partial N_{1}\right)_{z_{2}, V}$ of species 1 . It is, in fact, easy to see from Eq. (3) that

$$
\begin{aligned}
& p\left(N_{1}, z_{2}, V\right)=p_{2}\left(z_{2}\right)+\Pi\left(N_{1}, z_{2}, V\right), \\
& \mu_{1}\left(N_{1}, z_{2}, V\right)=\omega_{1}\left(z_{2}\right)+\widetilde{\mu}_{1}\left(N_{1}, z_{2}, V\right),
\end{aligned}
$$

with the osmotic pressure $\Pi \equiv-(\partial A / \partial V)_{N_{1}, z_{2}}$ and the shifted chemical potential $\tilde{\mu}_{1}\left(N_{1}, z_{2}, V\right) \equiv\left(\partial A / \partial N_{1}\right)_{z_{2}, V}$. As the shifts $p_{2}$ and $\omega_{1}$ are independent of density $\rho_{1}$ they do not influence the phase coexistence. ${ }^{9}$ Clearly, $\Pi$ and $\tilde{\mu}_{1}$ play the role of pressure and chemical potential in the onecomponent system interacting via $W$. (iii) It is interesting that the one-body term $\omega_{1}$ can be related to the Henry's law constant $h\left(z_{2}\right)=\lim _{\rho_{1} \rightarrow 0} \rho_{1} / z_{1}\left(\rho_{1}\right),{ }^{12}$ with $z_{1}$ the activity of species $1: z_{1}=\exp \left[\beta \mu_{1}\right] / \Lambda_{1}^{3}$. From Eq. (5) it follows that $h\left(z_{2}\right)=\exp \left[-\beta \omega_{1}\left(z_{2}\right)\right]$. The deviation of $h\left(z_{2}\right)$ from unity is a measure for the average 1-2 interaction, at given $z_{2}$, in the absence of 1-1 interactions. (iv) Although phase coexistence of the effective one-component system for a fixed temperature and given value of $z_{2}$ is completely determined by equality of osmotic pressures $\Pi$ and shifted chemical potentials $\widetilde{\mu}_{1}$ in two coexisting phases, one does require $p_{2}\left(z_{2}\right)$ and $\omega_{1}\left(z_{2}\right)$ explicitly in order to convert from $z_{2}$ to the average density $\rho_{2}=\left\langle N_{2}\right\rangle_{N_{1} z_{2} V} / V$ of particles of species 2 . This can be ascertained directly from the identity

$$
\left\langle N_{2}\right\rangle_{N_{1}, z_{2}, V}=-z_{2}\left(\partial \beta F / \partial z_{2}\right)_{N_{1}, V} .
$$

It is this conversion from the $\left(N_{1}, z_{2}, V\right)$ ensemble to the binary mixture $\left(N_{1}, N_{2}, V\right)$ ensemble that plays a crucial role in what follows. (v) The particular technique described here does not apply directly to Coulombic mixtures where other methods are required. ${ }^{7}$

\section{STRUCTURE AND ISOTHERMAL COMPRESSIBILITY}

In the previous section, we showed that a binary mixture can be mapped formally onto an effective one-component system with an effective interaction Hamiltonian $H^{\text {eff }}$, and corresponding potential of mean force $W$, by integrating out the degrees of freedom of one of the species in the partition sum and described how the phase behavior may be determined. Now we investigate the relation between structure and susceptibility (in this case the isothermal compressibility) in the binary mixture ensemble at fixed $\left(N_{1}, N_{2}, V\right)$ and the effective one-component ensemble at fixed $\left(N_{1}, z_{2}, V\right)$.
We begin by defining the $2 \times 2$ partial structure factor matrix in the binary mixture ensemble by

$$
S_{\alpha \beta}(k)=\frac{1}{\sqrt{N_{\alpha} N_{\beta}}}\left\langle\rho_{\alpha}(\mathbf{k}) \rho_{\beta}(-\mathbf{k})\right\rangle_{N_{1}, N_{2}, V},
$$

where $\rho_{1}(\mathbf{k})=\Sigma_{j=1}^{N_{1}} \exp \left[-i \mathbf{k} \mathbf{R}_{j}\right]$ and analogously for $\rho_{2}(\mathbf{k})$, and where the ensemble average of an observable $\mathcal{O}\left(N_{1}, N_{2} ;\{\mathbf{R}\},\{\mathbf{r}\}\right)$ is defined by

$$
\langle\mathcal{O}\rangle_{N_{1}, N_{2}, V}=\frac{\operatorname{Tr}_{1} \operatorname{Tr}_{2} \mathcal{O} \exp [-\beta H]}{\operatorname{Tr}_{1} \operatorname{Tr}_{2} \exp [-\beta H]} .
$$

It is a standard result that the $k \rightarrow 0$ limit of the partial structure factors $S_{\alpha \beta}(\mathbf{k})$ and the isothermal compressibility $\chi_{T}$ of the binary system are related by ${ }^{13}$

$$
\rho k_{B} T \chi_{T}=\frac{S_{11}(0) S_{22}(0)-S_{12}^{2}(0)}{x_{1} S_{22}(0)+x_{2} S_{11}(0)-2 \sqrt{x_{1} x_{2}} S_{12}(0)},
$$

where $\rho=\left(N_{1}+N_{2}\right) / V, x_{1}=N_{1} /\left(N_{1}+N_{2}\right)$, and $x_{2}=N_{2} /$ $\left(N_{1}+N_{2}\right)$, and where $\chi_{T}$ is defined as

$$
\chi_{T}^{-1}=-V\left(\frac{\partial p}{\partial V}\right)_{N_{1}, N_{2}},
$$

where $p$ denotes, as before, the pressure of the binary mixture and the temperature $T$ is, as usual, fixed.

In the effective one-component system at fixed $\left(N_{1}, V, z_{2}\right)$ the structure factor (of species 1 , of course) is defined by

$$
S^{\mathrm{eff}}(k)=\frac{1}{N_{1}}\left\langle\rho_{1}(\mathbf{k}) \rho_{1}(-\mathbf{k})\right\rangle_{N_{1}, V, z_{2}},
$$

where the ensemble average of an observable $\mathcal{O}\left(\{\mathbf{R}\}, N_{1}\right)$, i.e., an observable that is independent of $N_{2}$ and $\{\mathbf{r}\}$ and thus commutes with $\mathrm{Tr}_{2}$, is now given by

$$
\begin{aligned}
\langle\mathcal{O}\rangle_{N_{1}, V, z_{2}} & =\frac{\operatorname{Tr}_{1} \Sigma_{N_{2}=0}^{\infty} z_{2}^{N_{2}} \operatorname{Tr}_{2} \mathcal{O} \exp [-\beta H]}{\operatorname{Tr}_{1} \Sigma_{N_{2}=0}^{\infty} z_{2}^{N_{2}} \operatorname{Tr}_{2} \exp [-\beta H]} \\
& =\frac{\operatorname{Tr}_{1} \mathcal{O} \exp [-\beta W]}{\operatorname{Tr}_{1} \exp [-\beta W]} .
\end{aligned}
$$

Note that the potential of mean force $W$ can be replaced by $H^{\text {eff }}$ on the right-hand side of Eq. (12), since the difference gives rise to a common factor in the numerator and the denominator. The $k \rightarrow 0$ limit of the structure factor $S^{\text {eff }}$ of a one-component system of density $\rho_{1}$ and pressure $\Pi$ satisfies

$$
\rho_{1} k_{B} T \chi_{T, \text { eff }}=\lim _{k \rightarrow 0} S^{\text {eff }}(k),
$$

with the effective compressibility $\chi_{T \text {,eff }}$ defined as

$$
\chi_{T, \text { eff }}^{-1}=-V\left(\frac{\partial \Pi}{\partial V}\right)_{N_{1}, z_{2}} .
$$

The effective compressibility is therefore the osmotic compressibility. Clearly, Eq. (13) is the one-component analog of (9), and Eq. (14) that of (10).

We are now in a position to connect several of the quantities defined above. A key ingredient of this identification is 
the fact that any ensemble average $\langle\mathcal{O}\rangle$ of a (static) observable $\mathcal{O}\left(\{\mathbf{R}\}, N_{1}\right)$ is independent of the ensemble in the thermodynamic limit $N_{1} \rightarrow \infty, V \rightarrow \infty$ with $N_{1} / V$ constant. On the basis of Eqs. (7) and (11) we therefore have

$$
S_{11}(k) \equiv S^{\mathrm{eff}}(k),
$$

provided the thermodynamic state points are identical, i.e., provided $N_{2}=\left\langle N_{2}\right\rangle_{N_{1}, z_{2}, V}$. Together with $p=\Pi+p_{2}$, as defined in Eq. (4), and the definitions of the compressibilities in Eqs. (10) and (14), the identity of Eq. (15) directly yields

$$
\chi_{T}^{-1}=\chi_{T, \text { eff }}^{-1}-V\left(\frac{\partial z_{2}}{\partial V}\right)_{N_{1}, N_{2}}\left[\left(\frac{\partial \Pi}{\partial z_{2}}\right)_{N_{1}, V}+\left(\frac{\partial p_{2}}{\partial z_{2}}\right)\right] .
$$

The identification of the structure factor in Eq. (15) and the relation between the total and the osmotic compressibility in Eq. (16) are the central results of this article. The difference between $\chi_{T}$ and $\chi_{T \text {,eff }}$ involves the volume dependence of $z_{2}$ at fixed $N_{1}$ and $N_{2}$, and the dependence of both $\Pi$ and $p_{2}$ on $z_{2}$, i.e., not only properties of the effective one-component system, but also the thermodynamics of the reservoir of species 2 , at fugacity $z_{2}$.

If the structure factors are identical, it follows that the total pair correlation functions are also identical: $h_{11}(r)$ $\equiv h^{\text {eff }}(r)$. Moreover, it is straightforward to generalize to three-body, four-body, etc., correlations. These higher-order correlations will be identical, provided once again the state points are chosen to be identical. However, we wish to emphasize that (equilibrium) correlation functions of species 1 are only identical in the effective one-component system and the binary mixture, provided the exact potential of mean force $W$ is used in the determination of the statistical average Eq. (12). It is not guaranteed that an approximate mapping of the two-component system onto a one-component system will preserve this identity of the structure, even if the predictions for the phase behavior that result from the approximate mapping are reasonably accurate. A typical exact $W$ involves pair-, triplet-, and higher-body terms, while a typical approximate $W$ contains only pair interactions.

In the remainder of this article we study a special binary mixture for which the mapping onto an effective onecomponent system can be performed exactly and explicitly. This system is therefore especially well-suited to illustrate the main results of this section, embodied in Eqs. (15) and (16).

\section{ASAKURA-OOSAWA MODEL}

Reference 9 shows that the so-called Asakura-Oosawa model, which is a simple model for colloid-polymer mixtures, can be mapped exactly onto an effective onecomponent Hamiltonian with $W$ strictly pairwise, when the polymers are sufficiently small compared with the colloids. Here we calculate the structure factors and the pair correlation functions for the two-component Asakura-Oosawa model and those for the corresponding effective onecomponent system. We demonstrate explicitly from our numerical results that the structure factors, the pair correlation functions, and the isothermal compressibilities in the two systems are the same.
In the Asakura-Oosawa model the colloids are modeled as hard spheres with diameter $\sigma_{1}=\sigma_{11}$. The polymer coils are treated as interpenetrating, noninteracting particles as regards their mutual interactions $\left(\sigma_{22}=0\right)$. However, the polymers are excluded by a center of mass distance of $\sigma_{12}$ $=\left(\sigma_{1}+\sigma_{2}\right) / 2$ from the colloids, where $\sigma_{2}$, the diameter of the polymer coil, is given by $\sigma_{2}=2 R_{g}$ with $R_{g}$ the radius of gyration. The pairwise potentials in this simple model are thus given by

$$
\begin{aligned}
& \phi_{11}\left(\mathbf{R}_{i j}\right)= \begin{cases}\infty, & \left|\mathbf{R}_{i j}\right|<\sigma_{11}, \\
0, & \text { otherwise }\end{cases} \\
& \phi_{12}\left(\mathbf{R}_{i}-\mathbf{r}_{j}\right)=\left\{\begin{array}{ll}
\infty, & \left|\mathbf{R}_{i}-\mathbf{r}_{j}\right|<\sigma_{12} \\
0, & \text { otherwise }
\end{array},\right. \\
& \phi_{22}\left(\mathbf{r}_{i j}\right)=0 .
\end{aligned}
$$

Here $\mathbf{R}_{i}$ and $\mathbf{r}_{j}$ are the positions of the centers of the colloids and the polymer coils, respectively, while $\mathbf{R}_{i j}=\mathbf{R}_{i}-\mathbf{R}_{j}$ and $\mathbf{r}_{i j}=\mathbf{r}_{i}-\mathbf{r}_{j}$.

The structure of the homogeneous binary AsakuraOosawa model is characterized by three partial correlation functions $h_{i j}\left(r ; \rho_{1}, \rho_{2}\right)$ and three pairwise direct correlation functions $c_{i j}\left(r ; \rho_{1}, \rho_{2}\right)$ which satisfy the coupled OrnsteinZernike relations:

$$
h_{i j}(r)=c_{i j}(r)+\sum_{l=1}^{2} \rho_{l} \int c_{i l}\left(\left|\mathbf{r}-\mathbf{r}^{\prime}\right|\right) h_{l j}\left(r^{\prime}\right) d \mathbf{r}^{\prime} .
$$

In principle one could determine the $h_{i j}$ by computer simulation. However, for the very asymmetric situations of interest here, where the size ratio $q \equiv \sigma_{2} / \sigma_{1}$ is small, ergodic problems arise when the packing fraction of the small (polymer) species 2 becomes substantial. In order to avoid such difficulties, we solved Eq. (18) numerically using the Percus-Yevick (PY) closure relations

$$
\begin{array}{ll}
h_{i j}(r)=-1, & r<\sigma_{i j}, \\
c_{i j}(r)=0, & r>\sigma_{i j} .
\end{array}
$$

We chose to restrict our attention to very small values of the polymer packing fraction and fairly small values of the colloid packing fraction where the PY closure should be very accurate. Figures 1-5 show examples of the resulting partial correlation functions $h_{11}(r), h_{12}(r)$, and $h_{22}(r)$, and the corresponding partial structure factors $S_{i j}(k)$ for the fixed size ratio $q=0.15$, and for various state points, characterized by colloid and polymer packing fractions $\eta_{1}=\pi \sigma_{1}^{3} \rho_{1} / 6$ and $\eta_{2}=\pi \sigma_{2}^{3} \rho_{2} / 6$. As expected, the colloid-colloid structure becomes more pronounced as the colloid packing fraction $\eta_{1}$ increases at fixed $\eta_{2}$ : compare Figs. 2, 4, and 5. For $\eta_{1}$ $=0.30$ the colloid-polymer pair-correlation function $h_{12}(r)$ and structure factor $S_{12}(k)$ (see dotted lines in Fig. 5) also exhibit pronounced structure. Increasing $\eta_{2}$, the polymer fraction, at fixed $\eta_{1}$, leads to a rapid increase in the colloidcolloid $h_{11}(r)$ near contact $r=\sigma_{1}$ and to an increase in the polymer-polymer structure factor $S_{22}(k)$ at small $k$ : compare Figs. 1 and 2 and Figs. 3 and 4.

We now consider the structure and thermodynamics of the associated one-component system, and compare the re- 

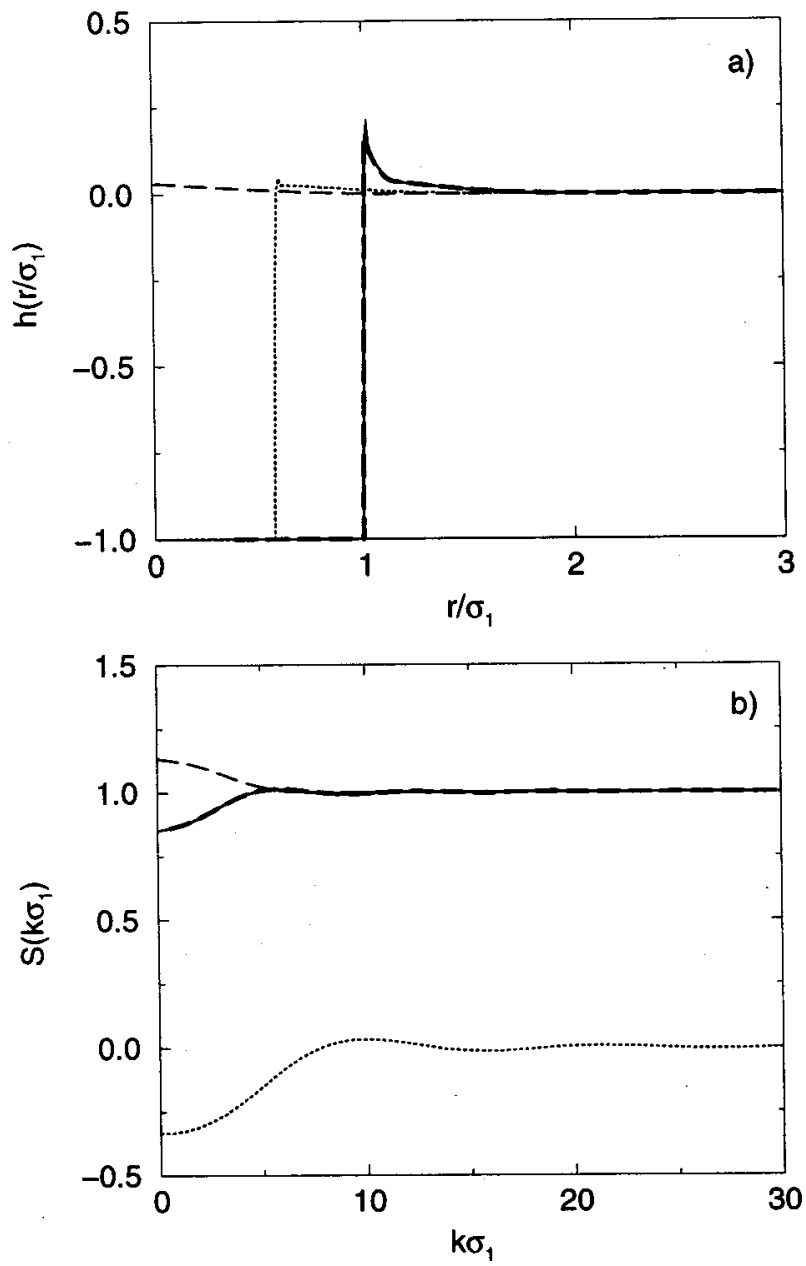

FIG. 1. Colloid-colloid (full line), colloid-polymer (dotted line), and polymer-polymer (dashed line) partial pair correlation functions $h\left(r / \sigma_{1}\right)$ (a) and structure factors $S\left(k \sigma_{1}\right)$ (b) for a colloid-polymer mixture with size ratio $q=0.15$, colloid packing fraction $\eta_{1}=0.02$ and polymer packing fraction $\eta_{2}=0.01$. The thick dashed line denotes the colloid pair correlation function (a) and structure factor (b) for the corresponding effective onecomponent system, based on the Asakura-Oosawa pair potential Eqs. (20) and (21).

sults with those of the binary mixture. It is well-known that three or more nonoverlapping large hard spheres cannot simultaneously overlap with a single smaller sphere, if the size ratio $q<0.1547 .{ }^{14}$ In Ref. 9, this was shown to imply that the $n$-body terms in the potential of mean force of Eq. (2) vanish, i.e., $\omega_{n} \equiv 0$, for $n \geqslant 3$. Thus, for these small size ratios, the only nonzero contributions to $W$ are the pairwise terms:

$$
W\left(N_{1}, z_{2} ;\{\mathbf{R}\}\right)=\sum_{i<j}^{N_{1}}\left[\phi_{11}\left(R_{i j}\right)+\omega_{2}\left(z_{2} ; R_{i j}\right)\right],
$$

where the bare pair potential $\phi_{11}$ is given in Eq. (17), and where $\omega_{2}$, the polymer-induced contribution to the pair potential, was derived by Asakura and Oosawa ${ }^{4}$
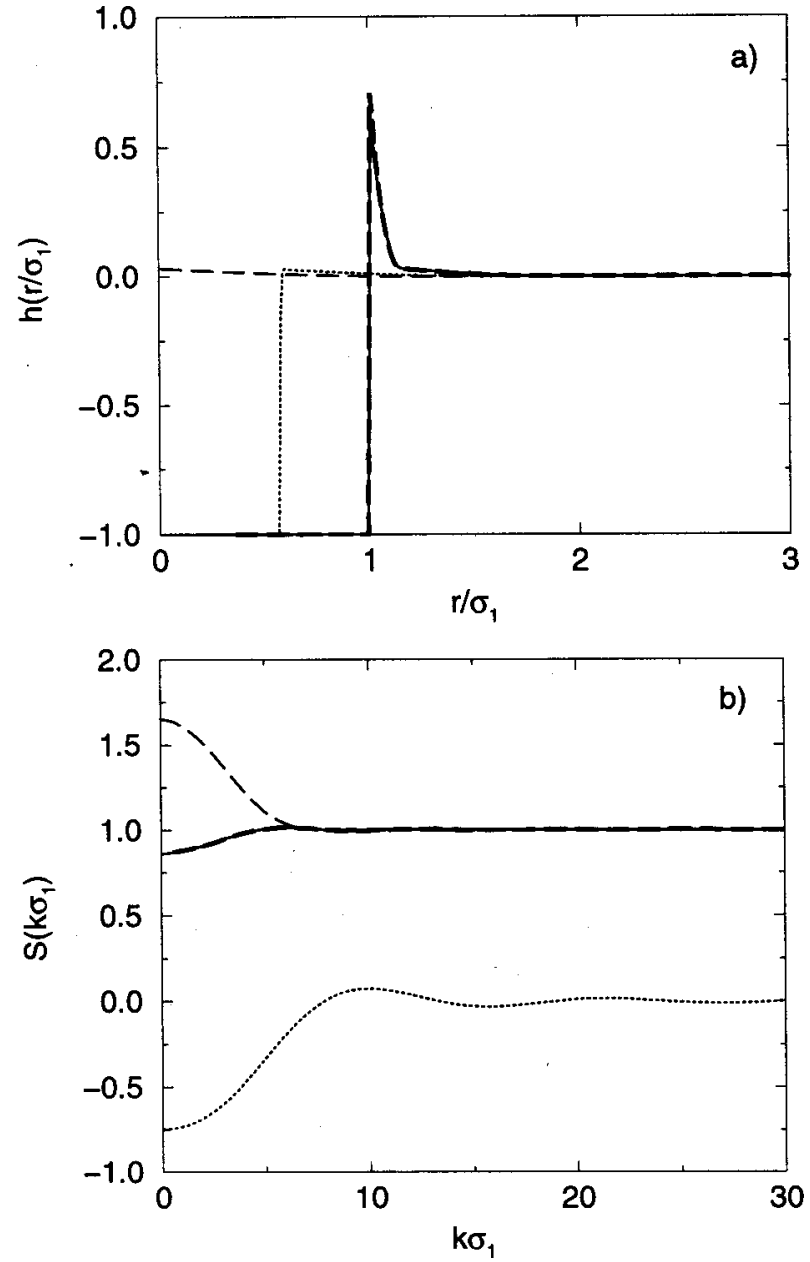

FIG. 2. As in Fig. 1 but now $\eta_{1}=0.02$ and $\eta_{2}=0.05$.

$\beta \omega_{2}\left(z_{2} ; R_{i j}\right)=\left\{\begin{array}{c}-\frac{\pi \sigma_{2}^{3} z_{2}}{6} \frac{(1+q)^{3}}{q_{3}} \\ \times\left[1-\frac{3 R_{i j}}{2(1+q) \sigma_{1}}+\frac{R_{i j}^{3}}{2(1+q)^{3} \sigma_{1}^{3}}\right], \\ \quad \text { for } \sigma_{1}<R_{i j}<\sigma_{1}+\sigma_{2},\end{array}\right.$

0 , otherwise.

This Asakura-Oosawa pair potential describes an attractive potential well close to the surface of the colloid, whose depth increase linearly with increasing $z_{2}$. The range of the potential is given by $\sigma_{2}$. Due to the ideal character of the polymers in this model, the one-body term $\omega_{1}$ and the reservoir pressure $p_{2}$ are also known exactly ${ }^{9}$

$$
\begin{aligned}
& \beta \omega_{1}\left(z_{2}\right)=z_{2} \frac{\pi}{6} \sigma_{1}^{3}(1+q)^{3}, \\
& \beta p_{2}\left(z_{2}\right)=z_{2}=\rho_{2}^{r}\left(z_{2}\right),
\end{aligned}
$$

where $\rho_{2}^{r}\left(z_{2}\right)$ is the density of ideal polymer in the reservoir. We have thus specified completely the effective onecomponent Hamiltonian of the Asakura-Oosawa model. Clearly the absence of higher-body contributions to $W$ leads to an enormous simplification, allowing us to have an ex- 

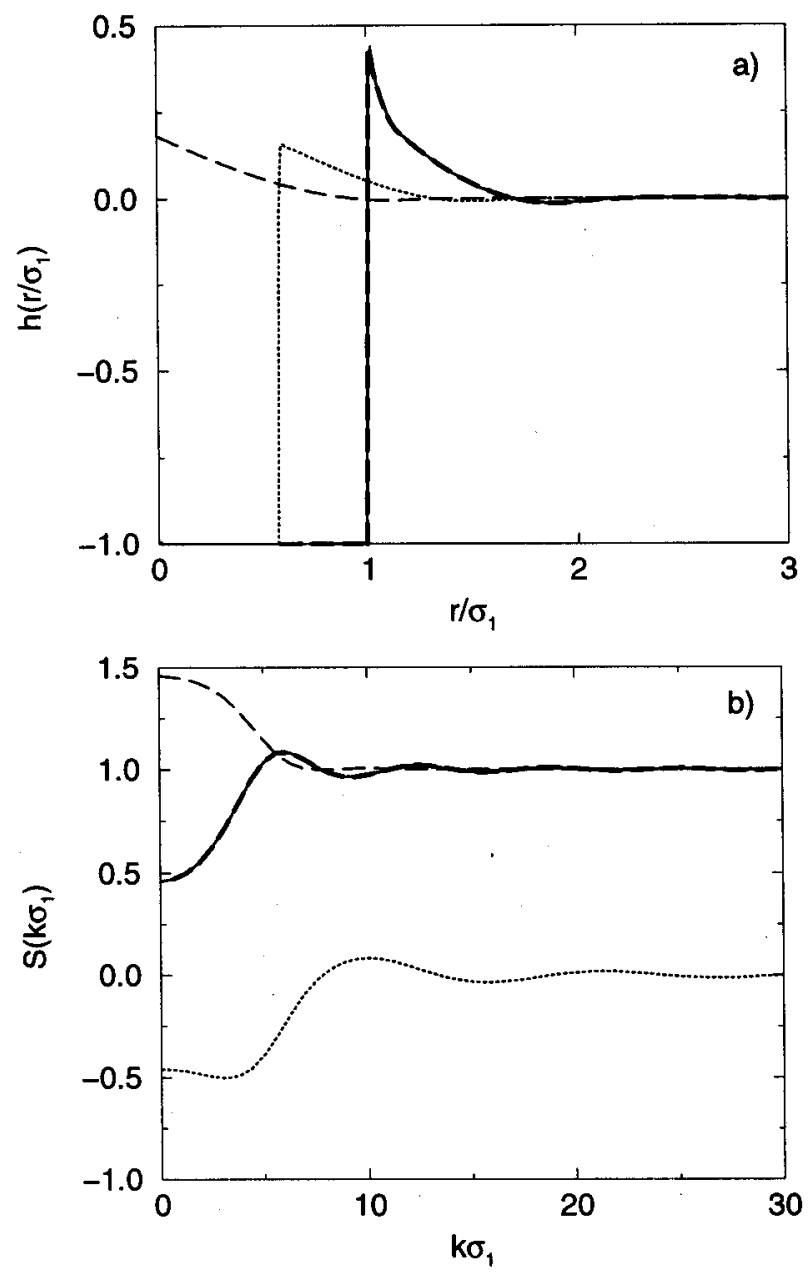

FIG. 3. As in Fig. 1 but now $\eta_{1}=0.10$ and $\eta_{2}=0.01$.

plicit effective Hamiltonian, which is, nevertheless, an exact representation of the original two-component model. Now we calculate the effective one-component pair correlation function $h^{\text {eff }}\left(r ; \rho_{1}, z_{2}\right)$, which is related to the effective onecomponent direct correlation function $c^{\mathrm{eff}}\left(r ; \rho_{1}, z_{2}\right)$ through the one-component Ornstein-Zernike equation

$$
h^{\mathrm{eff}}(r)=c^{\mathrm{eff}}(r)+\rho_{1} \int d \mathbf{r}^{\prime} c^{\mathrm{eff}}\left(\left|\mathbf{r}-\mathbf{r}^{\prime}\right|\right) h^{\mathrm{eff}}\left(r^{\prime}\right) .
$$

As in the binary mixture case we employ the PY closure, which now takes the form

$$
\begin{aligned}
& h^{\mathrm{eff}}(r)=-1, \quad r<\sigma_{11}, \\
& c^{\mathrm{eff}}(r)=\left(1-\exp \left[\beta \omega_{2}\right]\right)\left[h^{\mathrm{eff}}(r)+1\right], \quad r>\sigma_{11},
\end{aligned}
$$

where the numerical solution of Eqs. (23) and (24) then yields, for various state points $\left(\eta_{1}, z_{2}\right)$ and fixed size ratio $q=0.15$, the pair correlation $h^{\text {eff }}(r)$ and the structure factor $S^{\text {eff }}(k)=\left[1-\rho_{1} \hat{c}^{\text {eff }}(k)\right]^{-1}$.

In order to be able to compare the resulting $h^{\text {eff }}(r)$ and $S^{\text {eff }}(k)$ for given $\left(\eta_{1}, z_{2}\right)$ with the previous $h_{11}(r)$ and $S_{11}(k)$ obtained for given $\left(\eta_{1}, \eta_{2}\right)$, we need to convert between the
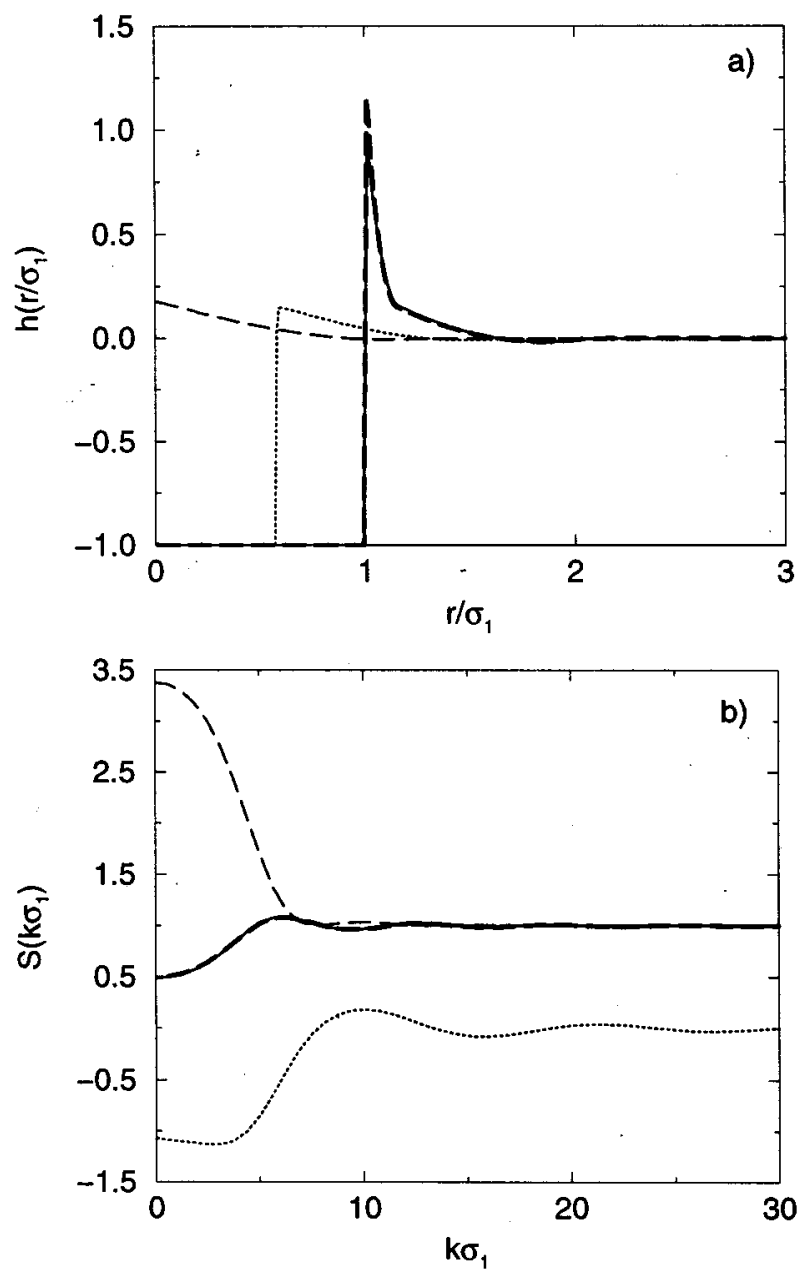

FIG. 4. As in Fig. 1 but now $\eta_{1}=0.10$ and $\eta_{2}=0.05$.

$\left(N_{1}, N_{2}, V\right)$ ensemble and the $\left(N_{1}, z_{2}, V\right)$ ensemble. This conversion is provided by Eq. (6), which with Eq. (3) can be rewritten as

$$
\begin{aligned}
\left\langle N_{2}\right\rangle_{N_{1}, z_{2}, V}= & z_{2} V \frac{\partial \beta p_{2}\left(z_{2}\right)}{\partial z_{2}}-z_{2} N_{1} \frac{\partial \beta \omega_{1}\left(z_{2}\right)}{\partial z_{2}} \\
& -z_{2}\left(\frac{\partial \beta A}{\partial z_{2}}\right)_{N_{1}, V} .
\end{aligned}
$$

For $q<0.1547$ and provided $\eta_{1}$ is not too high, it is an excellent approximation to neglect the third term on the right-hand side of Eq. (25). ${ }^{15}$ Then, with Eq. (22), we obtain

$$
\frac{\left\langle N_{2}\right\rangle_{N_{1}, z_{2}, V}}{V}=z_{2}\left[1-\eta_{1}(1+q)^{3}\right],
$$

or equivalently

$$
z_{2}\left(\eta_{1}, \eta_{2}\right)=\frac{6}{\pi \sigma_{2}^{3}} \frac{\eta_{2}}{1-\eta_{1}(1+q)^{3}} .
$$

Employing the conversion of Eq. (27), we can compare our results for the binary mixture with those of the effective onecomponent system. Note that Eq. (27) with (22) implies that 

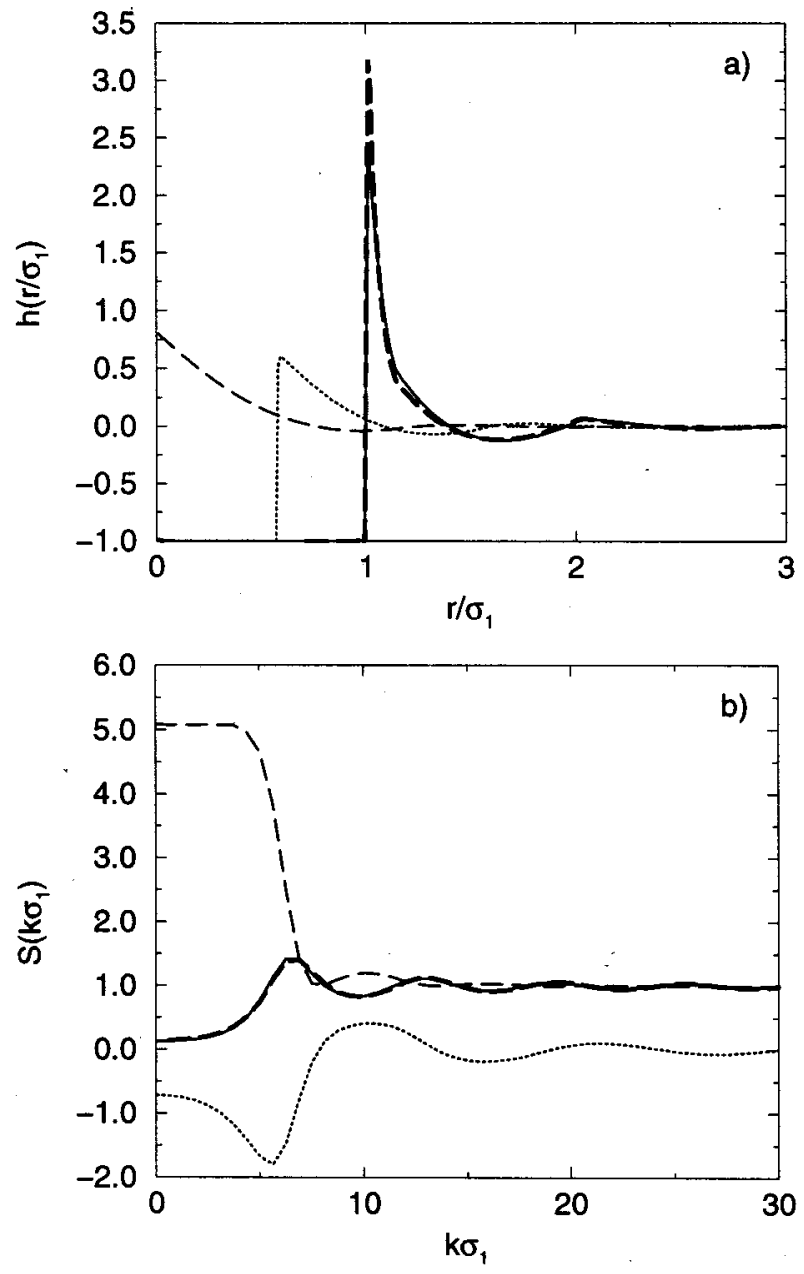

FIG. 5. As in Fig. 1 but now $\eta_{1}=0.30$ and $\eta_{2}=0.05$.

the ratio of polymer fraction in the binary mixture to that in the reservoir is given by $\eta_{2} / \eta_{2}^{r}\left(z_{2}\right)=1-\eta_{1}(1+q)^{3}$.

Alongside the pair correlation functions $h_{i j}(r)$ and the structure factors $S_{i j}(k)$ of the binary mixture at several state points, Figs. $1-5$ also display $h^{\text {eff }}(r)$ and $S^{\text {eff }}(k)$ of the corresponding one-component system, with $z_{2}$ determined from Eq. (27). It is evident that $S_{11}(k)=S^{\text {eff }}(k)$ and $h_{11}(r)$ $=h^{\mathrm{eff}}(r)$ within the accuracy of the plots in all five cases shown. This confirms that for this particular model the mapping is exact. Note that the comparisons in Figs. 1-5 all refer to very low polymer packing fractions, viz., $\eta_{2} \leqslant 0.05$, although the colloid fractions can be substantially higher, viz., $\eta_{1} \leqslant 0.30$. We deliberately restricted our consideration to this regime because of the approximate nature of the PY-closure employed in our calculations. It is important to recognize that the PY treatment for the two-component system is not precisely equivalent to $\mathrm{PY}$ for the effective one-component system. However, for sufficiently low packing fractions the differences between the two treatments should be negligible. For $\eta_{2}>0.05$ we do find that $h_{11}(r)$ begins to deviate significantly from $h^{\text {eff }}(r)$ (there is already an indication of small deviations in Fig. 5) but we emphasize that this reflects our use of the PY closure and not any fundamental failing of the mapping.

Within the one-component description it is easy to un-
TABLE I. Dimensionless isothermal compressibility $\rho k_{B} T \chi_{T}$ for the binary Asakura-Oosawa model obtained from the colloid-colloid structure factor $S_{11}(0)$, colloid-polymer structure factor $S_{12}(0)$, and polymer-polymer structure factor $S_{22}(0)$ and Eq. (9) for size ratio $q=0.15$ and varying colloid and polymer packing fractions $\eta_{1}$ and $\eta_{2}$.

\begin{tabular}{rccccc}
\hline \hline$\eta_{1}$ & $\eta_{2}$ & $S_{11}(0)$ & $S_{12}(0)$ & $S_{22}(0)$ & $\rho k_{B} T \chi_{T}$ \\
\hline 0.02 & 0.01 & 0.850 & -0.333 & 1.133 & 0.94 \\
0.02 & 0.05 & 0.860 & -0.748 & 1.656 & 0.94 \\
0.10 & 0.01 & 0.460 & -0.461 & 1.460 & 0.71 \\
0.10 & 0.05 & 0.483 & -1.070 & 3.380 & 0.72 \\
0.30 & 0.05 & 0.125 & -0.715 & 5.085 & 0.29 \\
\hline \hline
\end{tabular}

derstand why $h_{11}(r)\left[=h^{\text {eff }}(r)\right]$ increases near contact as more polymer is added at fixed colloid packing fraction. Increasing $\eta_{2}$ simply increases $-\omega_{2}\left(z_{2} ; \sigma_{1}\right)$, the well depth of the Asakura-Oosawa pair potential leading to enhanced attraction near contact; recall $h^{\mathrm{eff}}(r) \simeq \exp \left[-\beta \omega_{2}\left(z_{2} ; r\right)\right]-1$ for low colloid fractions.

Having confirmed that the colloid structure is the same in the binary and effective one-component systems we can investigate the isothermal compressibilities in the two corresponding systems. In the binary Asakura-Oosawa model, the isothermal compressibility can be calculated from the $k \rightarrow 0$ limit of the partial structure factors, using Eq. (9). The results for $S_{i j}(0)$ and for the dimensionless quantity $\rho k_{B} T \chi_{T}$ at the five states considered in Figs. 1-5 can be found in Table I. Recall that $\rho=\rho_{1}+\rho_{2}$.

It follows from the effective one-component expression for $\chi_{T}$ in Eq. (16), together with Eqs. (13) and (22), that

$$
\frac{1}{\rho k_{B} T \chi_{T}}=\frac{x}{S^{\mathrm{eff}}(0)}+\left(\frac{\partial z_{2}}{\partial \rho}\right)_{x}\left[1+\left(\frac{\partial \beta \pi}{\partial z_{2}}\right)_{\rho_{1}}\right],
$$

where $x=x_{1}=\rho_{1} / \rho$. The first term on the right-hand side follows directly from the calculation of the colloid structure factor, and the prefactor of the second term follows from Eq. (27). We estimate the remaining term on the right-hand side of Eq. (28), namely $\left(\partial \beta \Pi / \partial z_{2}\right)_{\rho 1}$, from the approximate one-component equation of state $\beta \Pi\left(\rho_{1}, z_{2}\right)=\beta \Pi_{\mathrm{HS}}\left(\rho_{1}\right)$ $+B_{2}^{\mathrm{AO}}\left(z_{2}\right) \rho_{1}^{2}$, with $\Pi_{\mathrm{HS}}$ the one-component hard-sphere pressure and $B_{2}^{\mathrm{AO}}$ the second virial coefficient associated with the Asakura-Oosawa pair interaction of Eq. (21). This yields $\left(\partial \beta \Pi / \partial z_{2}\right)_{\rho_{1}}=\rho_{1}^{2}\left[\partial B_{2}^{\mathrm{AO}}\left(z_{2}\right) / \partial z_{2}\right]$. We have now determined explicit expressions for all three terms on the righthand side of Eq. (28). All three contributions, as well as the resulting values of $\rho k_{B} T \chi_{T}$, are listed in Table II. Comparing the far right columns of Tables I and II confirms that the isothermal compressibility of the effective one-component system is equal to that of the corresponding binary AsakuraOosawa model for all five state points. Comparing the magnitudes of the three contributions on the right-hand side of Eq. (28) shows that the main contribution to the inverse isothermal compressibility is due to the second, ideal polymer term. Changing the volume of the effective one-component system at fixed $N_{1}$ and $N_{2}$ gives rise to a different fugacity $z_{2}$. It is this volume dependence of $z_{2}$ at fixed $N_{1}$ and $N_{2}$ (combined with the dependence of the polymer reservoir 
TABLE II. Dimensionless isothermal compressibility $\rho k_{B} T \chi_{T}$ obtained from Eq. (28) for states corresponding to those in Table I, calculated for the effective one-component system based on the Asakura-Oosawa pair potential Eqs. (20) and (21). $\eta_{2}^{r}$ is the packing fraction of polymer in the reservoir.

\begin{tabular}{cccccc}
\hline \hline & & $\frac{x}{S^{\mathrm{eff}}(0)}$ & $\left(\frac{\partial z_{2}}{\partial \rho}\right)_{x}$ & $\left(\frac{\partial z_{2}}{\partial \rho}\right)_{x}\left(\frac{\partial \beta \Pi}{\partial z_{2}}\right)_{\rho 1}$ & $\rho k_{B} T \chi_{T}$ \\
\hline 0.02 & $0.0103(0.01)$ & 0.0079 & 1.057 & $-9.2 e-5$ & 0.94 \\
0.02 & $0.0516(0.05)$ & 0.0016 & 1.062 & $-1.0 e-4$ & 0.94 \\
0.10 & $0.0118(0.01)$ & 0.0710 & 1.345 & $-2.9 e-3$ & 0.71 \\
0.10 & $0.0590(0.05)$ & 0.0138 & 1.382 & $-3.4 e-3$ & 0.72 \\
0.30 & $0.0920(0.05)$ & 0.1588 & 3.315 & $-8.0 e-2$ & 0.29 \\
\hline \hline
\end{tabular}

pressure $p_{2}$ on $z_{2}$ ) that mainly determines the inverse isothermal compressibility. The final term in Eq. (28) is $2 \%$ or $3 \%$ of the total for $\eta_{1}=0.30$ while for the first four states considered in Table II it is completely negligible. Thus, the particular choice of approximation for $\Pi\left(\rho_{1}, z_{2}\right)$ is not important in estimating this term. The first term, which corresponds to the inverse osmotic compressibility, contributes at the few percent level. Clearly it is not appropriate to approximate the compressibility of the true binary mixture $\chi_{T}$ by that of the effective one-component system, $\chi_{T \text {,eff }}$. In general the former can be expected to be much smaller than the latter, as was found in the single example studied by Louis et al. in Ref. 11. Those authors were not able to account for the large difference between their calculated values which, as we have seen, resides in the ideal polymer term, not considered in their work.

\section{DISCUSSION}

In this article we have argued that care should be taken when one compares thermodynamic properties of a binary mixture with those obtained from the corresponding effective one-component system. We first showed that, for exact mappings, the colloid-colloid structure in the mixture is equal to the colloidal structure in the effective one-component system, provided that the thermodynamic state points are identical, i.e., $N_{2}=\left\langle N_{2}\right\rangle_{N_{1}, z_{2}, V}$. We then showed that $\chi_{T}$, the isothermal compressibility of the mixture, is equal to the total compressibility of the corresponding effective onecomponent system, but should be carefully distinguished from $\chi_{T \text {,eff }}$, the osmotic compressibility of the effective onecomponent system, which is proportional to the $k \rightarrow 0$ limit of the structure factor of the colloids. The difference between the total and the osmotic compressibility involves the volume dependence of the fugacity $z_{2}$ at fixed particle numbers $N_{1}$ and $N_{2}$, and the dependence of the osmotic pressure $\Pi$ and the reservoir pressure $p_{2}$ on $z_{2}$. Explicit calculations for the Asakura-Oosawa model of a binary colloid and (ideal) polymer mixture with size ratio $q=0.15$ confirm that the structure of the colloids and the total compressibility are the same in the effective one-component system as in the mixture. Our results show that for the low packing fractions we consider $\chi_{T \text {,eff }}^{-1}$ makes only a small contribution to $\chi_{T}^{-1}$ and that it is the volume dependence of $z_{2}$ which makes the main contribution. There are clear repercussions for the compress- ibility of real polymer-colloid mixtures. Either one attempts to measure all three partial structure factors near $k=0$ and utilizes Eq. (9) (this is a tall order even with modern neutron scattering techniques) or one attempts to obtain $\chi_{T}$ from Eq. (16), making some assumption about $p_{2}\left(z_{2}\right)$ and the volume dependence of $z_{2}$.

We have, of course, ignored the atomic/molecular nature of the solvent. This leads to further complications in the interpretation of the actual compressibility of the ternary (colloid + polymer + solvent $)$ mixture. In principle one can include solvent effects using the present formalism. For example, for a one-component colloidal suspension the analysis of Secs. II and III remains valid and the compressibility of the suspension is given by Eq. (9), where 1 refers to colloid and 2 to solvent, or (assuming pairwise additive potentials for solvent and colloid) Eq. (16) where $p_{2}$ and $z_{2}$ refer to the reservoir of solvent. We are not aware of attempts to interpret the measured compressibility of suspensions in terms of Eq. (16) but we suspect they exist.

Finally, we conclude with some remarks about charged colloids. As mentioned in Sec. I experiments on chargestabilized colloids show that the osmotic bulk modulus as determined from the colloidal structure factor at long wavelengths [see Eq. (13)] is three times smaller than that calculated from a simple elastic theory using the potential of mean force $W$. It is important to realize that the latter quantity, which is based on $W$, yields again an osmotic bulk modulus, and thus a fair comparison is made in Ref. 10. In both approaches no attempt is made to make connection with the total bulk modulus of the ternary mixture of colloids and co-and counterions. However, the potential of mean force $W$ that is employed in their elastic theory contains only the repulsive DLVO pair interactions, while the exact $W$ may contain triplet, and higher-body interactions. The discrepancy between the osmotic bulk modulus obtained from the colloidal structure factor and the elastic theory can, thus, be explained by the approximation made in the potential of mean force and we therefore support their conclusions that the DLVO pair interactions alone cannot explain the results of the scattering experiments and that triplet, and higherbody interactions are nonnegligible.

Indeed recent theoretical studies of effective interactions in a charged-stabilized colloidal suspension show that a many-body cohesion can explain some of the features of the experimentally observed phase behavior. ${ }^{7}$ In this work, the three-component system of charged colloids, co-, and counterions is mapped onto an effective one-component colloidal system with an approximate effective Hamiltonian for the colloids that consists of a one-body term and purely repulsive DLVO pair interactions, which both depend nontrivially on the colloid density. In this approximate theory, the manybody cohesion is embodied in the density-dependent one body and pair terms. The result is the prediction of a gasliquid transition terminating in a critical point which is driven by the one-body term or "volume term" (Ref. 7). As the effective pairwise potentials are purely repulsive they cannot account for a critical point divergence of the colloid structure factor as $k \rightarrow 0$. On the other hand, the exact effective one-component Hamiltonian, with two-, three-, and 
more body interactions, would show the structure factor diverging when a critical point is approached. ${ }^{16}$ It is tempting to argue that in this approximate theory (parts of) the threeand higher-body terms are lumped together in the one body and pair terms, which thereby become nontrivially dependent on the colloid density. This will be investigated in a further study.

We should also mention a theoretical study on the influence of the nonzero size of the small ions on the structure factors of a micellar system by Klein et al. ${ }^{17}$ They show that, when the size of the small ions is sufficiently large, the partial structure factors of the micelles calculated from a primitive approach for the mixture differ significantly from the structure factor of an effective one-component system with a potential of mean force $W$ that contains only the DLVO potential for the micelles. The discrepancy between the micellar structure factor of the mixture and the effective onecomponent system can be accounted for by the approximation made in $W$. Triplet- and higher-body interactions are again nonnegligible when the micelles are not much larger than the ions.

\section{ACKNOWLEDGMENTS}

It is a pleasure to thank Ard Louis and Joe Brader for stimulating discussions. M.D. and R.v.R. thank Bristol University for hospitality during the initial stages of this work. This work was made possible by financial support under EPSRC Grant No. GR/L89013 and from the EPSRC Liquid
Matter Network and is part of the research program of the "Stichting voor Fundamenteel Onderzoek der Materie (FOM)," which is financially supported by the "Nederlandse Organisatie voor Wetenschappelijk Onderzoek (NWO)."

${ }^{1}$ W. G. McMillan and J. E. Mayer, J. Chem. Phys. 13, 276 (1945).

${ }^{2}$ E. J. W. Verwey and J. Th. G. Overbeek, Theory of the Stability of Lyotropic Colloids (Elsevier, Amsterdam, 1948).

${ }^{3}$ A. Vrij, Pure Appl. Chem. 48, 471 (1976).

${ }^{4}$ S. Asakura and F. Oosawa, J. Chem. Phys. 22, 1255 (1954).

${ }^{5}$ J. Bibette, J. Colloid Interface Sci. 147, 474 (1991).

${ }^{6}$ S. Hachisu, Y. Kobayashi, and A. Kose, J. Colloid Interface Sci. 42, 342 (1973); K. Tokano and S. Hachisu, ibid. 66, 130 (1978).

${ }^{7}$ R. van Roij and J. P. Hansen, Phys. Rev. Lett. 79, 3082 (1997); R. van Roij, M. Dijkstra, and J. P. Hansen, Phys. Rev. E 59, 2010 (1999).

${ }^{8}$ M. Dijkstra, R. van Roij, and R. Evans, Phys. Rev. Lett. 81, 2268 (1998); 82, 117 (1999); Phys. Rev. E 59, 5744 (1999).

${ }^{9}$ M. Dijkstra, J. M. Brader, and R. Evans, J. Phys.: Condens. Matter 11, 10079 (1999).

${ }^{10}$ J. A. Weiss, A. E. Larsen, and D. G. Grier, J. Chem. Phys. 109, 8659 (1998).

${ }^{11}$ A. A. Louis, R. Finken, and J. P. Hansen, Europhys. Lett. 46, 741 (1999).

${ }^{12} \mathrm{~T}$. L. Hill, in An Introduction to Statistical Thermodynamics (Dover, New York, 1986).

${ }^{13}$ J. P. Hansen and I. R. McDonald, Theory of Simple Liquids (Academic, London, 1986).

${ }^{14}$ A. P. Gast, C. K. Hall, and W. B. Russel, J. Colloid Interface Sci. 96, 251 (1983).

${ }^{15}$ J. M. Brader (private communication).

${ }^{16}$ P. B. Warren, J. Chem. Phys. 112, 4683 (2000).

${ }^{17}$ R. Klein, W. Hess, and G. Nägele, in Physics of Complex and Supermolecular Fluids, edited by S. A. Safran and N. A. Clark (Wiley, New York, 1987). 